\title{
Use of Direct Oral Anticoagulants for Treating Venous Thromboembolism in Patients With Cancer
}

Presented by Gerald A. Soff, MD

\section{Abstract}

For patients with cancer who experience venous thromboembolism (VTE), low-molecular-weight heparin (LMWH) remains the standard of care in the NCCN Guidelines for VTE, but under certain conditions direct oral anticoagulants (DOACs) are acceptable alternatives. A growing body of literature suggests that DOACs may more effective than LMWHs in preventing recurrences, but they do carry some increased risk of bleeding. Most of this risk is seen in patients with gastrointestinal or urinary pathology or implanted devices. DOACs are also acceptable when the pain, cost, and inconvenience of LMWHs are expected to be obstacles to compliance. Through careful patient selection, most patients can be treated successfully with a DOAC, although for most patients with gastrointestinal or urinary pathology, LMWH remains the safer choice.

J Natl Compr Canc Netw 2018;16(5.5):670-673 doi: 10.6004/jnccn.2018.0041

\section{Direct Oral Anticoagulants: Advances in Treatment and Prevention of Recurrent Thrombosis}

Depending on cancer type, up to $20 \%$ of patients will develop a venous thromboembolism (VTE) at some time during the disease course. ${ }^{1}$ The choice of appropriate anticoagulant in a given patient reflects a balance of efficacy and the risk of bleeding. Although low-molecular-weight heparin (LMWH) remains the standard of care for cancer-associated VTE, emerging new clinical data suggest that direct oral anticoagulants (DOACs) may be more effective in preventing recurrent episodes. ${ }^{2-4}$ However, 2 recent randomized studies reported an increased risk of bleeding with DOACs compared with LMWHs. ${ }^{3,4}$ Although the relative benefits and risks of DOACs versus LMWHs continue to be studied, the current NCCN Guidelines for Cancer-Associated VTE continue to recommend LMWHs for most patients. Exceptions are made for those who cannot or will not accept LMWH.

Presented by Gerald A. Soff, MD, Hematology Service, Memorial Sloan Kettering Cancer Center, New York, New York.

Dr. Soff has disclosed that he has received consulting fees/honoraria and grant/research support from Amgen Inc., and Janssen Pharmaceutical Products, LP.

Correspondence: Gerald A. Soff, MD, Hematology Service, Memorial Sloan Kettering Cancer Center, 1275 York Avenue, Howard-717, New York, NY 10065. Email: soffg@mskcc.org
At the NCCN 23rd Annual Conference, Gerald A. Soff, MD, Chief, Hematology Service, Memorial Sloan Kettering Cancer Center (MSKCC), and Professor of Clinical Medicine, Weill Cornell Medical College, presented data and addressed the rapidly evolving understanding of management of cancer-associated VTE. Dr. Soff presented data showing greater efficacy with DOACs compared with LMWHs, but cautioned about an increased risk of bleeding in patients with genitourinary tract pathology or nephrostomy tubes, or in patients with active gastrointestinal mucosal abnormalities such as duodenal ulcers, gastritis, esophagitis, or colitis. Genitourinary or genitourinary mucosal abnormalities, when exposed to a direct anticoagulant during absorption or excretion, are particularly susceptible to bleeding, and he recommended that a LMWH is preferable in those settings.

Based on his MSKCC cohort studies and recent randomized trials, Dr. Soff said the data are "very reassuring that, with proper patient selection, we're able to achieve high levels of efficacy, safety, and patient satisfaction" by treating cancer-associated VTE with DOACs. Referring to the practice at MSKCC, he indicated, "At this point, most of our patients are receiving rivaroxaban, except for those with contraindications." 
DOACs in Treating VTE

\section{LMWH Was a Sea Change}

In 2002, a practice-changing study with dalteparin, a LMWH, demonstrated it to be more effective and of comparable safety versus warfarin, leading to a sea change in the treatment of cancer-associated thrombosis. ${ }^{5}$ In the CLOT trial, dalteparin showed a failure rate of approximately $9 \%$ at 6 months, compared with $17 \%$ for warfarin, with no significant difference in major bleeds. "This provided great reassurance and [LMWH] became the standard of care," he said. However, he added that despite the improved efficacy, LMWH has not been shown to reduce mortality.

Real-life experience with LMWH has been burdensome. "The fact is that the quality of life on LMWH is really quite brutal," Dr. Soff indicated. "Even though it's a small-volume injection with a small needle, the drug is very painful for many patients; patients tell us they can handle the chemotherapy and its side effects, but the pain from the shots is killing them."

The financial toxicity of this class of drugs is also an issue for many patients. "There's a burden to the patients' well-being - a painful assault on their abdomens, as well as their pocketbooks," he remarked.

\section{Are DOACs Better Options?}

DOACs have recently become alternative treatment options. In November 2012, rivaroxaban was the first in this class approved by the FDA for treatment of VTE. Approval of other DOACs have followed: apixaban, edoxaban, and betrixaban, which are factor Xa inhibitors, and dabigatran, which is a pure thrombin inhibitor.

There are some important differences between DOACs and LMWHs. First, DOACs are directacting; they are small molecules that are absorbed orally and directly bind to the active site of the coagulation enzyme (factor Xa or thrombin). LMWHs are core proteins with glycosaminoglycan side chains. Technically, they are not anticoagulants, but are cofactors for the true anticoagulant, which is antithrombin III. LMWHs are not active in the absence of antithrombin.

Both DOACs and LMWHs are partially cleared in the urine, but DOACs do not require other factors to be effective, whereas LMWH, an indirect anticoagulant, requires antithrombin III, which is not usually present in the urine. In patients with urinary tract lesions or damage, this could lead to increased urinary tract bleeding with a DOAC, and is one of the reasons Dr. Soff recommends against its use in this setting. Similarly, biologically meaningful concentrations of DOACs are present in the gastrointestinal tract, particularly in the stomach and small intestine. Patients with upper gastrointestinal pathology, such as a tumor or ulcer, will be at increased risk of gastrointestinal bleeding.

This has led to the updated NCCN Guidelines to state that "DOACs have been associated with urinary and intestinal tract bleeding, and should be use with caution in patients with urinary or gastrointestinal tract lesions, pathology, or instrumentation."

\section{Differences Drive Selection of Drug}

The FDA approved the first 3 DOACs with no cancer-specific data to clarify safety and efficacy for this population. The studies did include small numbers of patients with cancer, but they were not representative of the broader population of patients with cancer. Although no detriment in efficacy or safety was shown among patients with cancer, the studies did not establish guidance for using DOACs in this population, Dr. Soff said.

To gather information to inform the use of DOACs in patients with cancer, Dr. Soff and his colleagues at MSKCC began using rivaroxaban in 2013 through a quality assessment initiative, with several important qualifiers (Figure 1). They recommended against rivaroxaban in patients with active gastrointestinal or urinary tract lesions. "This was not simply primary gastrointestinal or urinary tract cancers. It

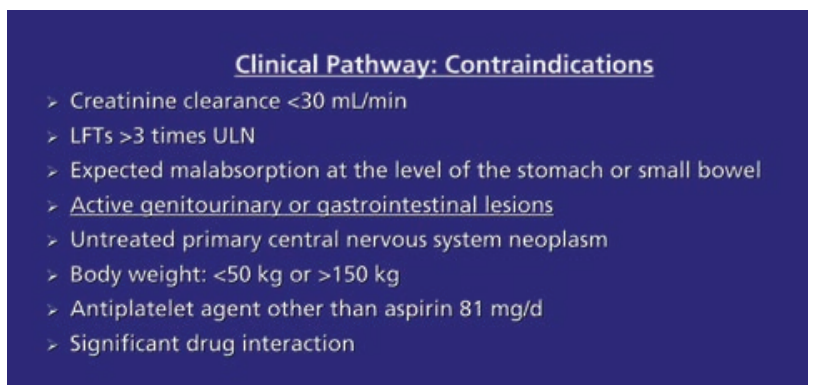

Figure 1. Rivaroxaban therapy for cancer-associated thrombosis: MSKCC institutional experience, 2014 to present. Abbreviations: LFTs, liver function tests; ULN, upper limit of normal. Data from Mantha S, Laube E, Miao Y, et al. Safe and effective use of rivaroxaban for treatment of cancer-associated venous thromboembolic disease: a prospective cohort study. J Thromb Thrombolysis 2017;43:166-171. 
could be someone who was post-gastrectomy, or had nephrostomy or urostomy tubes, any kind of 'hardware' in the urinary tract," he explained.

In 2017, the MSKCC group published the results on their first 200 patients with cancer-associated VTE who received rivaroxaban according to their inclusion criteria. ${ }^{2}$ They reported that recurrent VTE occurred in 4.4\%, major bleeding in 2.2\%, and clinically relevant nonmajor bleeding in 3.8\%; all-cause mortality rate was $17.6 \%$. As reference, in prior studies, LMWH has been associated with recurrent thrombosis in approximately $7 \%$ to $9 \%$ of patients and major bleeding in $6 \%$ to $7 \%{ }^{5,6}$ Dr. Soff cautioned, "This was not a prospective study, but a patient cohort. There are big differences in methodology between a clinical trial with an intentionto-treat analysis and real-world experience. But, we were at least encouraged that we were seeing no signs of inferior efficacy or safety."

Dr. Soff and colleagues have continued their quality assessment and, to date, >2,000 patients have been treated with rivaroxaban at MSKCC for cancer-associated thrombosis, central line clots, atrial fibrillation, and prophylaxis prior to orthopedic surgery. Of these, 1,075 patients had active cancer, many deemed at high risk for recurrence. In this cancer cohort, they have observed similar efficacy as in the 200-patient analysis: recurrent VTE in 4.2\%, major bleeding in $2.3 \%$, and clinically relevant nonmajor bleeding in $5.6 \%$, with all-cause mortality of $22.1 \%$ (unpublished data).

\section{Recent Clinical Trials}

At the 2017 American Society of Hematology (ASH) Annual Meeting, findings were presented from 2 clinical trials examining the efficacy of edoxaban and rivaroxaban in patients with cancer.

The Hokusai VTE Cancer trial was a randomized, open-label, noninferiority trial of LMWH versus the DOAC, edoxaban. All patients received dalteparin for at least 5 days. At randomization, patients continued on LMWH or transitioned to the DOAC. The dalteparin dose was $200 \mathrm{IU} / \mathrm{kg}$ daily for 1 month, followed by dalteparin $150 \mathrm{IU} / \mathrm{kg}$ daily; the edoxaban dose was $60 \mathrm{mg} / \mathrm{d}$. Treatment was given for at least 6 months and up to 12 months. ${ }^{3}$ For the primary outcome measure-a composite of recurrent VTE or major bleeding during the 12 months after randomization-edoxaban proved to be noninferior to LMWH (Figure 2).

"The hazard ratios for the composite end point were superimposable," Dr. Soff noted. There was a suggestion of greater efficacy with edoxaban, but with the consequence of a higher rate of major bleeding, mainly due to upper gastrointestinal bleeds in patients with primary gastrointestinal cancers. These findings speak to the need for proper patient selection, he emphasized.

A second randomized trial that compared rivaroxaban with dalteparin was presented at ASH. The Select-D trial, conducted in the United Kingdom, ${ }^{4}$ showed that 6 months of rivaroxaban was more effective than dalteparin at 6 months in reducing the risk of recurrent VTE, although it led to increases in major bleeds, clinically relevant bleeds, and total bleeds. Again, these tended to correlate with upper gastrointestinal cancers.

"It's very comforting and exciting that at least we see no evidence that switching from LMWH to DOAC causes a loss of efficacy. The studies so far have shown noninferiority of DOACs in efficacy, and some have shown a trend toward superiority," Dr. Soff said. "From past studies, it has been established that LMWH carries a 6-month recurrence rate of approximately $7 \%$ to $9 \%$. This risk is substantially lower with rivaroxaban, although for reasons that are unclear, in the edoxaban study, there was a fairly high recurrence rate. Edoxaban trended toward being more effective than LMWH, although this did not reach statistical significance."

In both of the trials presented at $\mathrm{ASH}$, the risk for bleeding was mostly in patients "who have an anatomic explanation for it," he added. "I think the critical thing for us going forward is patient selection. The question shouldn't be whether we use 1

\begin{tabular}{|l|c|c|c|c|}
\hline & $\begin{array}{c}\text { Edoxaban } \\
(\mathrm{N}=522)\end{array}$ & $\begin{array}{c}\text { Dalteparin } \\
(\mathrm{N}=524)\end{array}$ & $\begin{array}{c}\text { Hazard Ratio } \\
(95 \% \mathrm{Cl})\end{array}$ & P Value \\
\hline $\begin{array}{l}\text { Recurrent VTE or } \\
\text { major bleeding }\end{array}$ & $12.8 \%$ & $13.5 \%$ & $\begin{array}{c}0.97 \\
(0.70-1.36)\end{array}$ & $\begin{array}{c}.006 \\
\text { for noninferiority }\end{array}$ \\
\hline Recurrent VTE & $7.9 \%$ & $11.3 \%$ & $\begin{array}{c}0.71 \\
(0.48-1.06)\end{array}$ & .09 \\
\hline Major bleeding & $6.9 \%$ & $4.0 \%$ & $\begin{array}{c}1.77 \\
(1.03-3.04)\end{array}$ & .04 \\
\hline $\begin{array}{l}\text { Clinically relevant } \\
\text { nonmajor bleeding }\end{array}$ & $14.6 \%$ & $11.1 \%$ & $\begin{array}{c}1.38 \\
(0.98-1.94)\end{array}$ & \\
\hline
\end{tabular}

Figure 2. Clinical trial outcomes during the overall trial period. Abbreviation: VTE, venous thromboembolism.

Adapted from Raskob GE, van Es N, Verhamme P, et al. Edoxaban for the treatment of cancer-associated venous thromboembolism. N Engl J Med 2018;378:615-624. 
of the 4 DOACs versus LMWH, but rather in which patient should we be using which drug?"

\section{Anticoagulation Management for Invasive Procedures}

Bridging is no longer needed (treatment with warfarin had to be stopped 5-7 days before a procedure). With DOACs, 2 days off the drug before a procedure is usually sufficient for clearance. In patients who will receive spinal anesthesia, in elderly patients, and those with borderline renal function, clinicians should considering holding DOACs for 3 days, he advised, although there are no objective data to provide strong guidance.

\section{NCCN Guidelines}

The NCCN Guidelines for Cancer-Associated VTE state that 6 months of treatment with a LMWH is still preferred in patients with proximal VTEs and to prevent recurrences in those with advanced metastatic cancer. But for patients who refuse or have compelling reasons to avoid LMWH, DOACs are acceptable alternatives. Patients with urinary or gastrointestinal tract lesions, pathology, or instrumentation are probably best treated with LMWH. Patients without these conditions may be treated with a DOAC.

The language does provide a "get out of jail free card," Dr. Soff commented. "Patients may refuse or be poor candidates for LMWH because the injections are painful, inconvenient, and expensive. These factors may contribute to poor compliance with longterm LMWH treatment and support the choice of a DOAC." Studies have shown that fewer than half of the patients on LMWHs remain on prescribed treatment at 6 months. "If you factor in compliance and financial toxicity, there is more and more support that DOACs are an appropriate choice for the treatment of cancer-associated VTE," he stated.

\section{References}

1. Lyman GH. Preventing venous thromboembolism in cancer patients: can we do better? J Oncol Pract 2009;5:165-166.

2. Mantha S, Laube E, Miao Y, et al. Safe and effective use of rivaroxaban for treatment of cancer-associated venous thromboembolic disease: a prospective cohort study. J Thromb Thrombolysis 2017;43:166-171.

3. Raskob GE, van Es N, Verhamme P, et al. Edoxaban for the treatment of cancer-associated venous thromboembolism. N Engl J Med 2018;378:615624.

4. Young A, Marshall A, Thirlwall J, et al. Anticoagulation therapy in selected cancer patients at risk of recurrence of venous thromboembolism: results of the Select-D pilot trial [abstract]. Blood 2017;130:Abstract 625.

5. Lee AY, Levine MN, Baker RI, et al. Low-molecular-weight heparin versus a coumarin for the prevention of recurrent venous thromboembolism in patients with cancer. N Engl J Med 2003;349:146-153.

6. Smrke A, Gross PL. Cancer-associated venous thromboembolism: a practical review beyond low-molecular-weight heparins. Front Med (Lausanne) 2017;4:142.

7. Noble S, Prout H, Nelson A. Patients' xperiences of living with cancerassociated thrombosis: the PELICAN study. Patient Prefer Adherence 2015;9:337-345.

8. Khorana AA, McCrae KR, Milentijevic D, et al. Current practice patterns and patient persistence with anticoagulant treatments for cancerassociated thrombosis. Res Pract Thromb Haemost 2017;1:14-22. 